\title{
El conceplo moderno de Toxemia Gravídica
}

\author{
Por el doctor Miguel A. Fernández B.
}

Tema de gran importancia entre nosotros, en vísperas del primer Congreso Nacional de Obstetricia y Ginecología en cuyo temario está incluído; trataremos en el presente artículo de compendiar y aclarar numerosos conceptos al respecto, ya que en los últimos años, muchos de los clásicos ya han sido completamente revisados.

La frecuencia de aparición de las toxemias gravidicas, tomada de estadís ticas mundiales ${ }^{1}$ de 1930 a 1940, oscila para la colampsia entre $0,11 \%$ y $3,10 \%$; y entre $0,8 \%$ y $27,33 \%$ para las no convulsivas, correspondiendo las cifras más b.1jas a Holanda y Succia, y las más altas a la Indial y Pucrto Rico.

Al factor clima Dieckmann le ha dado gran importancia, scnalando la ma vor frecuencia de aparición en los estados del sur de los Estados Unidos, ase gurande que los climas húmedos son más propicios para la aparición de las coxemias, como sucede en Inglaterra ${ }^{1}$ en dende la mayor parte del ano el índice We humedad es mayor que en el reste de lauropa. Igualnente al factor raza tiene importancia, ya que en estadisticas obenidas en 1948 sobre 10.000 nacimientos listades (Inidos) se halló una frecuencia de casos de toxemia de $2,4 \%$ para la 1.1/a blanca, y $9.5 \%$ para otras razas.

La clasilicacion actualmente admitida, es la propuesta por Eastman I adoptada hoy por todos los autores de habla inglesa, que a más de ser sencilla es más precisa; divide las toxemias en: tosemias agudas y en toxemias crónicas o confermedad hipertensiva crónica; las primeras las subdividen en preclampsia y edampsia y las segundas en enfermedad hipertensiva crénica con o sin toxemia aguda sobreanadida.

El criterio para clasificar las agudas, se basa en el tiempo de aparición des. pués de las 24 semanas de embarazo, presien sistélicat de más de $140 \mathrm{~mm}$., dias télica de más de 90, edemas y proteinuria; el dato aislade de tensión alta debe persistir más de 24 horas para darle valor (Margaret llague. Ilosp.) o aún 48 foras (Chicago Lyining); una alza de 100 a 130 en presion sistrilica equivale a una de 130 a 150. La diferencia entre la preeclampsia y la eclampsia no es sino de intensidad, y se habla de la segunda, cuando aparece el cuadro convulsivo.

En cuanto a las toxemias crónicas o hipertensión esencial, no solamente se presentan en el embarazo, pero sí se agravan con él, haciéndose diagnosticables; 
los autores justifican su inclusión entre las toxicosis ${ }^{3}$, aduciendo que en muchos casos de mujeres embarazadas con hipertensión esencial, su diferenciación es imposible, y que por lo general gran parte de las hipertensiones de las multíparas tuvieron su origen en una toxicosis gravidica aguda sobrevenida en emba razos anteriores; pero la más funesta de las asociaciones tanto para la madre como para el feto es: enfermedad hipertensiva crónica con toxemia aguda. pues a más de la sintomatologia propia del cuadro agudo, cncontramos cambios retinianos graves, tales como hemorragias y edemas papilares, que nos explican la aprori ción de los sintomas funcionales visuales, de que se yucjom estas confermis; su aparición es precoe y se hace alrededor de las veinticuatro a treinta semanass !) tal manera que el criterio para clasificar una pacience dentro de la enfermedad hipertensiva vascular crónica con toxemia aguda sobre-anadida se basat: en los antecedentes de enfermedad hipertensiva anterior al embarazo, a la existencia de una elevación en la presión sistólica de $30 \mathrm{~mm}$. o más y diastólica de $15 \mathrm{~mm}$. o más sobre la cifra normal, y a la aparición de una marcada proteinuria. Por medio del laboratorio y valiéndonos de la determinación simultánea de pruebas de depuración urcica y de ácido úrico (urea clearance $y$ uric acid clearance) ${ }^{5}$ se puede establecer un diagnóstico diferencial entre la precelampsia, la enfermedad hipertensiva y la enfermedad renal; en la precelampsia la depuración de ácido úrico esta desproporcionadamente disminuida; ambas pruebas de depuración son normales en la enfermedad hipertensiva no complicada; y ambas bajas en la enfermedad renal. De tal manera que resumiendo: el término toxeniia aguda está solamente reservado a la precelampsia y la eclampsia, no siendo aplicable a ninguna otra clase de toxemia que no encuadre dentro de esta clasili cación, se puede en cambio hablar de toxemia de mediana o ligera intensidad sin prejuzgar sobre determinida entidad. La hiperemesis gravidica ha sido omi tida en la nucva clasificación, por la razón de que, todas las entidades anterior mente señaladas tienen un factor que les es commin: el factor vascular; siendo demostrado que en la hiperemesis ${ }^{3}$ no interviene dicho factor, ni coinciden tam poco (entre sí) los hallazgos anatomo patologicos, ni las pruchas de bioguímica patológica, ni menos alun los signes clinicos; al respecto Dickmann opina que "incluir la hiperemesis dentro de las toxemias, es no solamente ilógico sino que ayuda a hacer más conlusa la comprensión de este grupo de enfermedades" A las nefritis agudas, las glomeruloncfritis, nefrosis, y otras enfermedades renales inflamatorias o metabilicas, se les excluye igualmente, sin querer afirmar con esto que no se presenten en la mujer embarazada.

En relación con tratamiento, está demostrado desde hace algún tiempo que la profilaxia por medio de un adecuado control prenatal es de importancia ca pital. El dato de tensión y el uranálisis para incestigar albúmina es por todos practicado y debe hacerse cadal quince dias en les primeros seis meses, y cada semana en lostres ultimos", dele instruirse a las pacientes sobre los sintomas de Ta eclampsia, para yue consulien oportunamente si éstes se presentaren, dar gran importancia a la reducetom en la ingestión de cloruro de sodio con Ta alimentación, instituyendo una dieta debidamente balanceada en vitaminas y minerales $y$ de un índice calórico suficiente; hacer igualmente un control riguroso de peso, 
prara en caso de que el aumento sea exilgerado, estar solore aviso de una posible relención acuosa aun antes de ser perecptible la aparicion de edemas; tener especial cuidado en los casos ya demostrades de hipertension esencial crónica, dia-

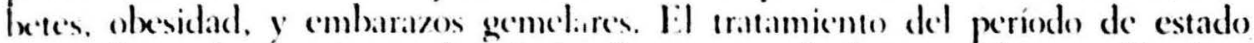
base, llenar los siguientes fines ${ }^{3}$ I. Prevencion de las convulsiones. Il--Preancion de la hipertension residual. III.-Parte de un foto vion, y IV.-Parto logrede con el minimo de trammatismo: los cuatro puntos unicamente se logran

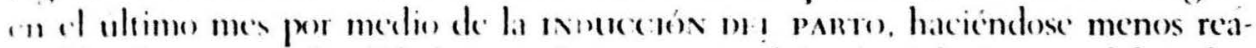
hrobles las cuatro finalidades, conforme se val alcjando del termino del emba1.120. la espera sistematica en los cases no viables no es recomendable ya que en I. e estadisticas del Hospital John Hopkins del ano 192t a 42 bubo 2.418 casos de toxemia en observación ${ }^{3}$ de los cuales 92 llegaron a ataque convulsivo, du- rantc la observación, con siete muentes maternas y 30 mortinatos por "contemporización". El tratamiento llamado imbulatorio, solamente se debe emplear en unfermas con ligeras alzas tensionalds (por debajo de $135 \mathrm{~mm}$. y $85 \mathrm{~mm}$.) sin colema y sin proteinuria; se utiliza ef sulfato de magnesia por vía oral, el reposo Y la dieta de dos gramos de cloruro de sodio, siendo controladas dichas enfermas der veces por semana; en caso de que con este tratamiento no se normalice ni el p $x$ ni los datos tensionales se le impone a la enferma la inmediata hospitaliza 14m. El criterio para hospitalizar una paciente se forma teniendo en cuenta ${ }^{6}$ : I". una tensión sistolica de $140 \mathrm{~mm}$. o más y diastolica de $90 \mathrm{~mm}$. o más; $2^{\circ}$, una notoria proteinuria $(+2 \mathrm{gr}$. al cateterismo $)$ y $3 \%$, aumento repetido y creciente de peso por encima de los limites normales. La historia clínica dele llevar iii detallado examen físico y un récord diario de los principales síntomas, tales coumo cefalea, disturbios visuales, edema palpelbral y maleolar, peso diario, tensiin tomada cada cuatro horas, "record" de ingesta y excreta de agua, examen hario de orina cuantitatio para albúmina y cilindros, examen de fondo de ofu el dia de admision y cada tercer dia según los hallazges patologicos, química ungunca (NPN, proténa total del sueros, ácido urico, contenido de dióxido the carbono) igualmente dato de hematocrito (normal 36\% a 47\%); debe te merse cuidado con las dosis demasiado repetidas de morlina y demás narcóticos por tener éstos un marcado poder antidiurético demostrado en experiencias llevidas a cabo en embarazadas normales, de tal manera que si los liquidos excretades disminuyen notablemente el uso de esta clase de drogas está contraindicado, pudiendo hacerse la sedación sin mayor peligro por nedio de los barbitúricos.

Bowes da cl siguiente espuema de conducta: a) Pacientes con tensión de 140 90, ligero edema, y trazas de alhumina en la orina: quictud, funcionamien (1) intestinal correcto, y dicta sin sal, induccion en case de que esta situación no se modifique en veinticuatro horas y el feto sea viable. b) Entermas con tenwion de 150 100, edema mis notorio y moderada albuminuria: se les prescribe yuitud en cama, dieta sin sal y vigilancia permanente en caso de presentar ce. falea, disturbios isuales o poca eliminación de liquidos; si la presión diastólica persiste alta, y la albuminuria no disminuye a pesar del tratamiento correcto, se debe inducir el trabajo para evitar la eclampsia y prevenir la muerte del feto. 
c) Pacientes con preeclampsia severa, con tensión superior a 180/110: se les debe dar liquidos ( $v$ ia iv), morfina o mejor fenobarbital; si no se presenta una mejoría marcada se debe inducir, siendo en algunos casos la cesárea una conducta que se impone en especial si la paciente es una primipara añosa. d) Si la paciente presenta la primera convulsión, el tratamiento de emergencia debe ser hecho inmediatamente; este consiste en aislamiento riguroso de la enferma, tra tando de evitar todo estímulo exterior, aspirar el vomito y mucosidades, y tomar las precauciones debidas para evitarle cualquicer tramiatismo; la observación continua es importante; una dosis incial de morfina de 16 mgrs., seguida hora y media despues de una inyecion intramuscular de sulfate de magnesia, 40 s. s. al $15 \%$ (en casos apurados dele inyectarse lentamente vía endovenosa), mor fina repetirla dos horas después de la priméra dosis, y media hora más tarde 4 grs. de sulfato de magensia, aplicar luego dos inyecciones más de 3 grs. cada una, 11 y media y 19 y media horas después de iniciado el tratamiento; el uso del cloruro de calcio 10 c. c. vía endovenosa en solución al $5 \%$ es de utilidad en estos casos para tratar de neutralizar el efecto tóxico producido por una dosis alta de sulfato de magnesia; por último, y solamente cuarenta y ocho horas después de haber cesado las convulsiones debe ser inducida la paciente. La conducta de extraer el feto por cesárea no debe ejecutarse sino en primiparas vie jas que no hayan tenido más de una o dos convulsiones.

En relación con la conducta por cesárea debemos anadir ${ }^{9}$ que hoy está completamente admitido, no practicarla sino cuando existe una indicación obstétrica, o cuando el cuello no permite un parto inmediato por vía vaginal; como quiera que ésta (la cesárea) no garantiza la cesación inmediata de las convul. siones.

El tratamiento por medio del veratrum, últimamente utilizado por Assali con compuestos más puros y más fácilmente dosificables que los antiguos ${ }^{10}$, em plea dosis de 0,2 c. c. de droga (veratrone Parke D).) cada hora en solución de glucosa al $5 \%$ en agua, vía endovenosa muy lenta. Las últimas experiencias publicadas en Estados Inidos, hechas siguiendo el método de Assali"1, dan las siguientes conclusiones: diez casos de preeclampsia severa y eclampsia tratados con veratrum (veratrone P. D.) vía endovenosi, ocho de estos tratados por otros métodos sin mejoria clínica, presentaron una mejoría notoria con baja de la tensión y desaparición de los sintomas en veinticuatro horas; los fenómenos de intolerancia tales como náuscas, vomito y bradicardia se presentaron en las pacientes en tratamiento, pero de poea intensidad y duración; las dosis usadas fucron: una inicial de 0,2 c. c. diluido en 1 c. c. de glucosa al $5 \%$, aplicado endo venoso lentamente (tres minutos de duración); la primera caida de la tensión sobrevino cinco minutos después, y ésta baja brusca duró cuarenta y cinco a sesenta minutos despucis de los cuales subió lentamente; las dosis subsiguientes se aplicaron por la misma via y a la misma solución, pero en forma de gota a gota cndovenoso, calculando inyectar 0,2 por hora; la excresión urinaria disminuyó por la baja brusca de tensión en el glomerulo, pero esto sucedió solamente en los primeros estadios, pues el cálculo de la eliminación en 24 horas fue satisfactorio, posiblemente por aparecer más tarde una poliuria compensadora. Los au- 
weres señalan como punto importante el hecho de haler critado toda sedación a las enfermas así tratadas. Debemos señalar por último, que el veratrum sólo obra como hipotensor en la embarazada toxémica, al contrario del cloruro de tetractilamonio que obra como hipotensor en las no toxémias ${ }^{10}$, dicho fenómeno poedemos aclararlo diciendo que en la embarazada normal la presión arterial es ussenida por un factor neurogénico muy sensible al cloruro de tetractilamonio 1 mus selectivo a la anestesia espinal pero prácticamente inefectivo al veratrum an combio en la cmbarazada con toxemia, la hipertension es mantenida especholmente por factores humorales, no afectados por el cloruro de tetractilamonio, pero si muy sensibles a la acción del veratrum².

\section{SUIMARIO:}

Se trata de hacer un resumen lo más completo, sobre los nuevos conceptos que prevalecen hoy en el estudio de las toxemias gravidicas.

Se explica la nueva clasificación adoptada hoy por todos los tratadistas de habla inglesa.

Trátanse de puntualizar las bases de esta clasificación y la forma de lograr un diagnóstico preciso de cada una de ellas.

Se aclaran conceptos sobre tratamiento de las toxemias, y se esboza un plan i seguir en la conducta de los distintos grados de toxemia haciéndose un comentario sobre la conducta quirúrgica.

Por último se trata de explicar la farmacodinamia del veratrum y la forma en que hoy se está utilizando en algunos hospitales norteamericanos.

\section{BIBLOGRAFIA:}

1. Dieckmann w. J. The Toxemias of Pregnancy, 39-39. St. Louis, 1952. C. B. Mosby.

2. Greenhill J. P. Toxemias. The 1950 Year Book of Obs. and Ginec., 109-110. August 1949, July 1950.

3.--Eastman N. J. Obstetries, 1950, 27-645. Appleton.

4.-Fernández-Bastidas M. A. Las Toxemias. Conferencia dictada en la Clinica Santa Lucía de Bogotá, marzo, 1952.

5. Chesley Leon C. Am. J. Obs. and Ginec. 59: 950-969. May 50.

Chesley L. C. Simultaneous renal clearence of urea and uric acid in differential diagnosis of late toxemias. Year Book of Obs. and Ginec., 112, 1950.

6. - Coscrove S. Margaret. Hague Mat. Hosp. Doctor's Guide. August 10/49.

7. -Brown W. E. Hodges and Bradbury. Study of antidiuretic effect of depressant drugs used in eclampsia. Am. J. Obs. and Ginec. 60: 1-18, July 1950.

8. - Bowes Kenneth. Recognition and Treatment of Toxemias of Pregnancy Practitioner, 166-124, 132, Feb. 1951.

9.- Posner Charles et Col. Harlem Hop. Bull. 4: 1-18. Jun 1951. Citado por Year Book of Obst. and Ginec. 1952.

10. Cincinnati. Hosp. Studies Related to Treatement of Toxemia of Pregnancy. Am. J. Obs. and Ginec. 60-315-323, August 1950.

11.-Alban E. J. et Col. Intravenous Veratrum Viride in the Treatement fo Toxemia. Am. J. Obs. and Ginec., 64-1.084-1.092, November 1952.

12.-Dieckmann w. J. The Toxemias of Pregnancy. 178. St. Louis, 1952. C. V. Mosby. 